\title{
Aberrant hepatic TRIB3 gene expression in insulin-resistant obese humans
}

\author{
H. Oberkofler • A. Pfeifenberger • S. Soyal • T. Felder • \\ P. Hahne $\cdot$ K. Miller $\cdot$ F. Krempler $\cdot$ W. Patsch
}

Received: 4 March 2010 /Accepted: 7 April 2010 /Published online: 13 May 2010

(C) Springer-Verlag 2010

\begin{abstract}
Aims/hypothesis The pseudokinase tribbles homologue 3 (Drosophila) (TRIB3) negatively interferes with insulinmediated phosphorylation and activation of v-akt murine thymoma viral oncogene homologue 1 (AKT1, also known as protein kinase B). Animal studies have shown that Trib3 expression was higher in the fasting state and in animal models of diabetes, promoting hyperglycaemia presumably by increasing glucose production in the liver. Less is known about the role of TRIB3 in insulin resistance in humans, although a gain-of-function mutation associated with abnormalities related to insulin resistance has been described in TRIB3.

Methods We determined hepatic mRNA expression of TRIB3 and selected genes encoding enzymes, transcription factors and coactivators involved in glucose homeostasis. We also determined biochemical variables of intermediary metabolism in obese patients with varying degrees of insulin resistance.
\end{abstract}

Electronic supplementary material The online version of this article (doi:10.1007/s00125-010-1772-2) contains supplementary material, which is available to authorised users.

H. Oberkofler $(\bowtie) \cdot$ A. Pfeifenberger $\cdot$ S. Soyal $\cdot$ T. Felder $\cdot$

P. Hahne $\cdot$ W. Patsch

Department of Laboratory Medicine,

Landeskliniken and Paracelsus Private Medical University Salzburg,

Müllner Hauptstr. 48,

A-5020 Salzburg, Austria

e-mail: h.oberkofler@salk.at

K. Miller

Department of Surgery, Krankenhaus Hallein,

Hallein, Austria

F. Krempler

Department of Internal Medicine, Krankenhaus Hallein,

Hallein, Austria
Results In our study population hepatic TRIB3 mRNA expression was associated with surrogate markers of insulin resistance. TRIB3 expression was significantly increased in a subgroup with high HOMA of insulin resistance (HOMA-IR) compared with a low HOMA-IR group $(p=0.0033)$. TRIB3 transcript levels were correlated with $P E P C K$ (also known as $P C K 2)$ mRNA expression $(p=0.0014)$ and mRNA expression of PPARGC1A $(p=0.0020)$, PPARGC1B $(p<0.0001)$, USF1 $(p=0.0017), F O X O 1 \quad(p=0.0003)$ and SREBP-1c (also known as $S R E B F 1 ; p=0.0360)$. Furthermore ligands of peroxisome proliferator-activated receptor $\alpha /$ retinoid $\mathrm{X}$ receptor and overexpression of its coactivator PPARGC1A as well as overexpression of $S R E B P-1 C$ and its coactivator PPARGC1B increased TRIB3 promoter activity in HepG2 cells.

Conclusions/interpretation We have found evidence for a role of aberrant hepatic TRIB3 transcript levels in insulin resistance in obese humans and identified potential transcriptional pathways involved in regulation of TRIB3 gene expression in the liver.

Keywords Gene expression - Insulin resistance · Transcription · Tribbles 3

\begin{tabular}{|c|c|}
\hline \multicolumn{2}{|c|}{ Abbreviations } \\
\hline AKT1 & $\begin{array}{l}\text { V-akt murine thymoma viral oncogene } \\
\text { homologue } 1\end{array}$ \\
\hline CRP & C-reactive protein \\
\hline HOMA-IR & HOMA of insulin resistance \\
\hline PPARA & Peroxisome proliferator-activated receptor $\alpha$ \\
\hline PPARGC1A & $\begin{array}{l}\text { Peroxisome proliferator-activated receptor } \gamma \\
\text { coactivator } 1 \text { alpha }\end{array}$ \\
\hline PPARGC1B & $\begin{array}{l}\text { Peroxisome proliferator-activated receptor } \gamma \\
\text { coactivator } 1 \text { beta }\end{array}$ \\
\hline SREBP & Sterol regulatory element binding protein $1 \mathrm{c}$ \\
\hline RIB3 & Tribbles homologue 3 \\
\hline
\end{tabular}




\section{Introduction}

Tribbles homologue 3 (TRIB3), the mammalian homologue of Drosophila tribbles, is classified as a pseudokinase with a truncated kinase domain that lacks ATP-binding and catalytic residues. An inhibitory role for TRIB3 in insulin signal transduction has been suggested, as it was shown to bind to the pleckstrin homology domain of the insulinresponsive Ser-Thr kinase, v-akt murine thymoma viral oncogene homologue 1 (AKT1), thereby preventing its membrane association, $\mathrm{Thr}^{308}$ phosphorylation and activation via upstream kinases [1, 2]. Although, in mice, deletion of Trib3 did not produce major disturbances in insulin signalling and glucose homeostasis [3], and in cultured rat hepatocytes adenoviral Trib3 overexpression failed to affect insulin mediated AKT1 phosphorylation [4], several other studies have reported data consistent with a role for Trib3 in insulin resistance. Trib3 expression was increased in livers of $d b / d b$ mice, a rodent model of type 2 diabetes [1]. Furthermore hepatic Trib3 gene knock-down enhanced insulin sensitivity, whereas adenovirus-mediated hepatic overexpression of Trib3 promoted hyperglycaemia and glucose intolerance in wild-type mice [5]. In the fasted state, TRIB3 was increased, thereby preventing residual insulin signalling and promoting glucose output by the liver [1]. Furthermore, increased expression of Trib3 has been implicated in ethanol-induced hepatic insulin resistance [2]. Most recently, a protein termed adaptor protein, phosphotyrosine interaction, $\mathrm{PH}$ domain and leucin zipper containing 1, which mediates adiponectin signalling, was shown to enhance insulin-stimulated activation of AKT1 and suppression of gluconeogenesis by blocking the interaction of AKT1 with TRIB3 through direct competition [6].

In humans, associations of a gain-of-function TRIB3 Glu84Arg missense polymorphism with phenotypes related to insulin resistance have been described [7]. Furthermore, overexpression of the variant Arg84 allele enhanced inhibition of insulin-mediated AKT1 phosphorylation in HepG2 cells [7] and was associated with impaired insulin signalling and nitric oxide production in human endothelial cells [8]. Because of a possible role of TRIB3 in insulin resistance in humans, we measured hepatic mRNA expression of TRIB3 in obese patients with varying degrees of insulin resistance and determined associations with biochemical variables and hepatic transcript levels of genes involved in transcriptional regulation and glucose homeostasis.

\section{Methods}

The study included 93 obese patients undergoing weightreducing surgery. Of these, 54 underwent a gastric banding procedure and 39 a gastric bypass procedure, in both cases under general anaesthesia (propofol and remifentanil). Participants were included if they had fasting blood glucose levels $<7.0 \mathrm{mmol} / \mathrm{l}$, no history of diabetes or use of glucoseor lipid-lowering medication, and no weight changes $>3 \%$ during the previous 2 months. All study participants provided informed consent and study protocols were approved by the local Ethics Committee. Adipose tissue mass, diameter of abdominal subcutaneous adipose tissue and visceral adipose tissue, and grading of fatty liver disease were determined as described [9]. Tissue biopsies were obtained from liver as well as visceral and subcutaneous adipose tissue at $\sim 20 \mathrm{~min}$ after induction of anaesthesia in patients undergoing gastric banding procedure and at $\sim 60 \mathrm{~min}$ in those undergoing gastric bypass, respectively; biopsies were collected in RNA-later (Ambion, Austin, TX, USA).

Plasma glucose, insulin, cholesterol, triacylglycerol, HDL-cholesterol and C-reactive protein (CRP) were determined as described [10]. Plasma adiponectin was measured using an immunoassay (Human Adiponectin/Acrp30; R\&D Systems, Wiesbaden-Nordenstadt, Germany). HOMA-IR was calculated as described previously [9]. Total RNA was isolated and reverse-transcribed as described [10]. Transcript levels of genes listed in Electronic supplementary material (ESM) Table 1 were quantified in duplicate using TaqMan gene expression assays (Applied Biosystems, Warrington, UK) and a real-time PCR detector (iCycler iQ Multicolour; Bio-Rad, Hercules, CA, USA). Constitutively expressed acidic ribosomal protein $\mathrm{p} 0$ mRNA was measured for normalisation of mRNA abundance.

The TRIB3-Prom-Luc plasmid was generated using 5'GAGACG CGTTGCAGGGATACAAAGCTCGTGA-3' ( -3073 to -3051$)$ and $5^{\prime}$-AGACTCGAGAGT ACCTCG CCCCGTCGTTCC-3' (489 to 510) as forward and reverse primers, respectively, to amplify a 3,583 bp DNA fragment that was cloned into the pGL3-Basic Vector (Promega, Madison, WI, USA) using restriction endonucleases MluI and XhoI. Numbers in parentheses refer to primer positions relative to the transcriptional start site (GenBank accession number NM_021158). The PPARGC1A (also known as $P G C-1 \alpha$ ), PPARGC1B (also known as $P G C-1 \beta$ ) and nuclear active $S R E B P-1 c$ expression constructs have been described previously [10].

Human HepG2 hepatoma cells were grown and transfected as described [10]. Unless otherwise indicated, we used $1 \mu \mathrm{g}$ of reporter plasmid, $0.5 \mu \mathrm{g}$ of expression plasmids and $1 \mathrm{ng}$ of pRL-TK plasmid (Promega) as transfection control. Drugs at concentrations of $10 \mu \mathrm{mol} / \mathrm{l}$ for retinoic acid and WY 14.643 were added immediately before transfection. Cells were collected $24 \mathrm{~h}$ after transfection, and firefly and Renilla luciferase activities were measured as described [10]. 
Table 1 Associations of hepatic TRIB3 mRNA with transcript levels of selected transcription factors, coactivators and genes involved in hepatic gluconeogenesis

\begin{tabular}{lll}
\hline Transcript & Regression coefficient (SE) & $p$ value \\
\hline PPARGC1A & $0.309(0.097)$ & $0.0020^{\mathrm{a}}$ \\
PPARGC1B & $0.451(0.091)$ & $0.000003^{\mathrm{a}}$ \\
SREBP-1c & $0.225(0.105)$ & 0.0360 \\
USF1 & $0.316(0.097)$ & $0.0017^{\mathrm{a}}$ \\
FOXO1 & $0.362(0.096)$ & $0.0003^{\mathrm{a}}$ \\
PEPCK & $0.323(0.098)$ & $0.0014^{\mathrm{a}}$ \\
\hline
\end{tabular}

Values are adjusted for age, sex and HOMA-IR, $n>85$ participants

${ }^{a}$ Significant after the Bonferroni correction

Differences in continuous variables between HOMA of insulin resistance (HOMA-IR) groups were ascertained by analysis of variance. To meet the equal variance and normality assumptions of analysis of variance, logarithmic transformations for plasma triacylglycerol, CRP, and hepatic and adipose tissue transcript levels were done. We adjusted measurements, by multiple regression for concomitant effects of sex and age. Categorical data were summarised by frequencies and analysed by the $\chi^{2}$ test. To estimate associations between hepatic TRIB3 mRNA levels and other hepatic transcripts levels or biochemical variables, we used multivariate linear regression. Logarithmic transformation of transcript variables was used to fulfil general linear model assumptions. Reported $p$ values are two-tailed. To test the significance of multiple comparisons, the Bonferroni correction was used.

\section{Results and discussion}

A detailed description of clinical and biochemical variables of our obese study population, characterised by varying degrees of insulin resistance, is shown in ESM Table 2. Among variables commonly associated with insulin resistance, sex and age-adjusted hepatic TRIB3 mRNA levels showed associations with plasma insulin $(p=0.0031)$, HOMA-IR $(p=0.0029)$, plasma triacylglycerol $(p=0.0089)$ and hepatic steatosis score $(p=0.0087)$. An inverse association was observed with plasma adiponectin $(p=0.0345$; ESM Table 3). However, the associations with steatosis score, adiponectin or plasma triacylglycerol were not maintained after adjustment for HOMA-IR. No associations were noted with plasma glucose, HDL-cholesterol and CRP or obesity indices such as BMI and fat mass (data not shown).

To elucidate the role of TRIB3 in insulin resistance in humans, we studied possible associations of hepatic, visceral and subcutaneous tissue transcript levels of TRIB3 in subgroups of 32 obese participants with HOMA-IR $<2.0$ and of 32 obese participants with HOMA-IR >5.0. The number of participants undergoing gastric banding or gastric bypass surgery was similar in the high- and lowHOMA-IR groups (ESM Table 4). Participants included in the high HOMA-IR group had significantly higher values for triacylglycerol and lower values for HDL-cholesterol, adiponectin and both visceral and subcutaneous SLC2A4 mRNA expression (ESM Table 4). Hepatic AKT1 phosphorylation, as determined by western blot analysis, was significantly reduced in participants with a high HOMA-IR $(p<0.05$; ESM Fig. 1). The sex- and age-adjusted comparison showed higher hepatic TRIB3 mRNA levels in the high HOMA-IR group than in the low HOMA-IR group ( $p=$ 0.0033; ESM Table 4). This difference remained significant
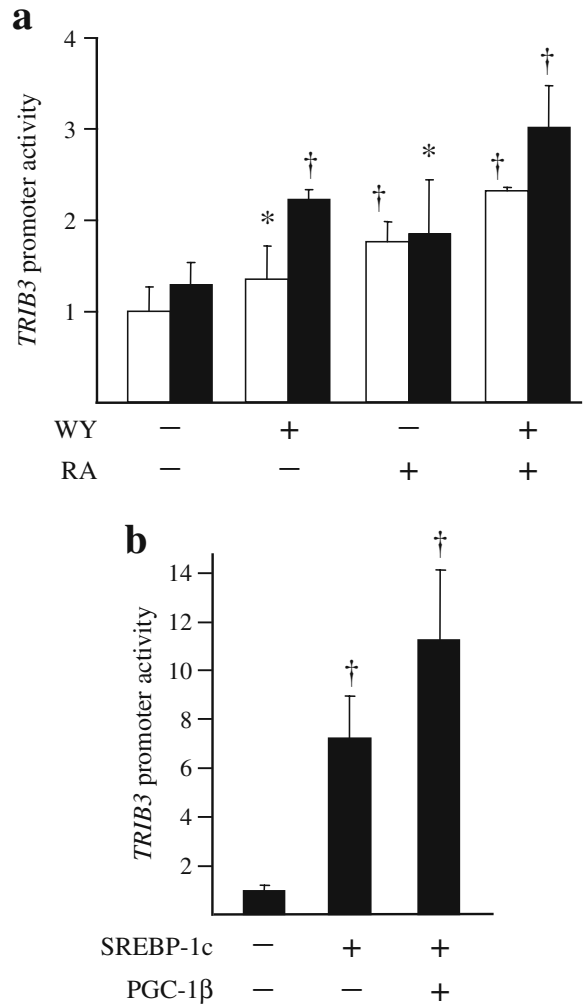

Fig. 1 PPAR $\alpha /$ retinoic $X$ receptor, SREBP-1c and their coactivators PPARGC1A and PPARGC1B respectively, are involved in the transcriptional regulation of human TRIB3 gene expression in HepG2 cells. a HepG2 cells were transiently transfected with the TRIB3Prom-Luc reporter plasmid or co-transfected with a human PPARGC1A (also known as $P G C-1 \alpha$ ) expression construct in the absence or presence of WY14.643 (WY) and retinoic acid (RA; $10 \mathrm{nmol} / 1$ each) as indicated. ${ }^{*} p<0.05$ and ${ }^{\dagger} p<0.005$ for basal $(-)$ compared with drug-stimulated $(+)$ reporter gene activities in the absence (white bars) or presence (black bars) of PPARGC1A overexpression. b HepG2 cells were transfected with TRIB3-Prom-Luc or co-transfected with SREBP-1c and/or PPARGC1B (also known as $P G C-1 \beta$ ) expression constructs as indicated. Basal TRIB3-Prom-Luc reporter gene activity in the absence of expression plasmids or ligands was set to 1.0. Data are presented as fold change $\pm \mathrm{SD} ; * p<0.05$ and ${ }^{\dagger} p<0.005$ 
after adjustment for the surgical procedure $(p=0.0053$; ESM Table 4). In addition, increased TRIB3 transcript abundance was also observed in visceral tissue samples ( $p=$ 0.0029). A similar trend, albeit not significant, was obtained in subcutaneous tissue specimens.

Sex-specific analysis in the larger group of women also showed higher TRIB3 mRNA levels in the high HOMA-IR group $(2.62 \pm 2.23)$ than in the low HOMA-IR group $(1.15 \pm$ $1.05 ; p=0.0195)$. A similar trend was observed in the smaller group of men $(p=0.1612)$. No significant interaction between sex and TRIB3 mRNA was observed. Hepatic TRIB3 expression displayed a strong correlation with PEPCK (also known as PCK2) mRNA expression (Table 1), which is consistent with previous observations demonstrating that adenoviral mediated overexpression of TRIB3 resulted in increased glucose output from the liver [1]. Our data obtained in humans are therefore consistent with a role for TRIB3 in insulin resistance in obese humans.

To gain insight into the transcriptional networks involved in the regulation of TRIB3 expression in humans, we measured hepatic mRNA levels of selected transcription factors and coactivators. In our study population, TRIB3 transcript levels, adjusted for age, sex and HOMA-IR, correlated with PPARGC1A, PPARGC1B, USF1 and FOXO1 mRNA expression levels, as well as, unexpectedly, with transcripts encoding sterol regulatory element binding protein 1c (SREBP-1c; Table 1). No associations were observed with PPARA, LXRA (also known as NRIH3), USF2, CHREBP (also known as MLXIPL) and CEBPA transcripts (data not shown). Previous studies in mice showed that Trib3 transcription is increased via coactivation of peroxisome proliferator-activated receptor $\alpha$ (PPARA) by peroxisome proliferator-activated receptor $\gamma$ coactivator 1 alpha (PPARGC1A, also referred to as PGC- $1 \alpha$ ) [5]. To determine whether transcription from the human TRIB3 promoter is activated by a similar mechanism, we transiently transfected HepG2 cells with a luciferase reporter construct driven by the human TRIB3 promoter (TRIB3-Prom-Luc). Incubation of transfected cells with PPARA and/or retinoic X receptor ligands (WY 14.643 and 9-cis retinoic acid, respectively) increased reporter gene activity, which was further augmented upon co-transfection with a PPARGC1A expression plasmid (Fig. 1a). Hence, PPARGC1A coactivates the PPARA-mediated transcription of the human TRIB3 gene.

A strong association was observed between TRIB3 mRNA and transcript levels of the transcriptional coactivator peroxisome proliferator-activated receptor $\gamma$ coactivator 1 beta (PPARGC1B, also referred to as PGC-1 $\beta$, which, in contrast to PPARGC1A, has been shown to bind and directly co-activate SREBPs [11]. Despite a modest association between TRIB3 transcripts and transcripts encoding SREBP-1c, recent studies have shown that nuclear import of SREBP-1c is enhanced by endoplasmic reticulum stress, which is implicated in insulin resistance [12]. We therefore directly determined effects of SREBP-1c and PPARGC1B on transcriptional regulation of the human TRIB3 gene, in co-transfection experiments using HepG2 cells. The TRIB3-Prom-Luc construct was transiently transfected together with a nuclear active SREBP-1c expression plasmid. Nuclear active SREBP-1c increased the basal transcriptional activity and coexpression of PPARGC1B further enhanced the SREBP-1c-mediated activation of the TRIB3-Prom-Luc reporter construct (Fig. 1b), indicating a role for SREBP-1c and PPARGC1B in the regulation of human TRIB3 gene transcription.

Components of the insulin-signalling pathway have been extensively studied over the past years in an attempt to identify their physiological contributions to the regulation of glucose homeostasis. TRIB3 is an inhibitor of the insulin-responsive AKT1 kinase and has been implicated in insulin resistance in several studies using cell lines and animal models $[1,2,5,6]$. In the present study, we provide initial evidence for an aberrant hepatic expression of TRIB3 in insulin-resistant human study participants. These results should, however, be interpreted with caution, as this phenomenon might be restricted to the obese population. Correlations of TRIB3 transcript levels with mRNA levels of distinct transcription factors and coactivators, together with our observations from cell culture, may help to unravel the underlying complex transcriptional pathways that result in human insulin resistance.

Acknowledgements This study was supported by grants from the Fonds zur Förderung der wissenschaftlichen Forschung (FWF, Project P19893B05), the Wiener Wissenschafts-, Forschungs-und Technologiefonds (WWTF Projects L507-058), the Land Salzburg and the Verein für Medizinische Forschung Salzburg, Austria.

Duality of interest The authors declare that there is no duality of interest associated with this manuscript.

\section{References}

1. Du K, Herzig S, Kulkarni RN, Montminy M (2003) TRB3: a tribbles homolog that inhibits Akt/PKB activation by insulin in liver. Science 300:1574-1577

2. He L, Simmen FA, Mehendale HM, Ronis MJ, Badger TM (2006) Chronic ethanol intake impairs insulin signaling in rats by disrupting Akt association with the cell membrane. Role of TRB3 in inhibition of Akt/protein kinase B activation. J Biol Chem 281:11126-11134

3. Okamoto H, Latres E, Liu R et al (2007) Genetic deletion of Trb3, the mammalian Drosophila tribbles homolog, displays normal hepatic insulin signaling and glucose homeostasis. Diabetes 56:1350-1356

4. Iynedjian PB (2005) Lack of evidence for a role of TRB3/NIPK as an inhibitor of PKB-mediated insulin signalling in primary hepatocytes. Biochem J 386:113-118 
5. Koo SH, Satoh H, Herzig S et al (2004) PGC-1 promotes insulin resistance in liver through PPAR-alpha-dependent induction of TRB-3. Nat Med 10:530-534

6. Cheng KK, Iglesias MA, Lam KS et al (2009) APPL1 potentiates insulin-mediated inhibition of hepatic glucose production and alleviates diabetes via Akt activation in mice. Cell Metab 9: 417-427

7. Prudente S, Hribal ML, Flex E et al (2005) The functional Q84R polymorphism of mammalian Tribbles homolog TRB3 is associated with insulin resistance and related cardiovascular risk in Caucasians from Italy. Diabetes 54:2807-2811

8. Andreozzi F, Formoso G, Prudente S et al (2008) TRIB3 R84 variant is associated with impaired insulin-mediated nitric oxide production in human endothelial cells. Arterioscler Thromb Vasc Biol 28:1355-1360
9. Felder T, Hahne P, Soyal SM et al. (2010) Hepatic adiponectin receptors (ADIPOR) 1 and 2 mRNA and their relation to insulin resistance in obese humans. Int $\mathrm{J}$ Obes (Lond). doi:10.1038/ ijo.2010.7

10. Oberkofler H, Hafner M, Felder T, Krempler F, Patsch W (2009) Transcriptional co-activator peroxisome proliferator-activated receptor (PPAR) gamma co-activator-1beta is involved in the regulation of glucose-stimulated insulin secretion in INS-1E cells. J Mol Med 87:299-306

11. Lin J, Yang R, Tarr PT et al (2005) Hyperlipidemic effects of dietary saturated fats mediated through PGC-1beta coactivation of SREBP. Cell 120:261-273

12. Kammoun HL, Chabanon H, Hainault I et al (2009) GRP78 expression inhibits insulin and ER stress-induced SREBP-1c activation and reduces hepatic steatosis in mice. J Clin Invest 119:1201-1215 